Bronchiolitis bei Kindern

\section{Weniger Symptome mit hypertoner Saline}

Die akute virale Bronchiolitis ist die häufigste Infektion der unteren Atemwege bei Kindern. Auslöser sind meist RespiratorySyncytial-oder Adenoviren. Eine kausale Therapie gibt es aber nicht, denn eine klinische Wirksamkeit für antivirale Substanzen ließ sich bislang nicht eindeutig nachweisen, die Gabe von Antibiotika ist nur bei einer bakteriellen Superinfektion sinnvoll. Die Behandlung beschränkt sich daher auf unterstützende Maßnahmen.

Eine effektive Therapieoption ist die Behandlung mit hypertoner Saline. So belegt ein Cochrane-Review nach Auswertung von vier randomisierten kontrollierten Studien (Zhang Let al. 2008) die Wirksamkeit einer Inhalation mit 3\%iger Saline allein oder in Kombination mit Bronchodilatatoren bei 254 Kindern (Alter $\leq 24$ Monate) mit akuter Broncholitis. Gegenüber 0,9\%iger Saline hatten Kinder nach Inhalation der vernebelten 3\%igen Saline einen signifikant niedrigeren Symptomscore.

\section{Milderer Krankheitsverlauf, kürzere Hospitalisierung}

Die Ergebnisse einer aktuellen Studie mit 93 hospitalisierten Kindern ( $\varnothing$ Alter: 5,8 $\pm 4,4$ Monate) die an milder bis moderater Bronchiolitis litten, bestätigen dieWirksamkeit der Inhalationstherapie mit vernebelter hypertoner Saline (z.B.MucoClear ${ }^{\circledR} 3 \%$ ). Die jungen Patienten erhielten dreimal täglich eine Inhalation mit 2,5 mg Salbutamol in isotonischer (0,9\%, Kontrolle) oder $3 \%$ iger Saline. Die Behandlung mit vernebelter 3\%iger Saline senkte Husten, Wheezing und Atemgeräusche signifikant schneller als in der Kontrollgruppe. Nach der Inhalation an Tag 1, 2 und 3 sank in der Studiengruppe auch der klinische Symptomscore schneller. In der Folge konnten Kinder, die vernebelte $3 \%$ ige Saline inhaliert hatten, auch signifikant schneller aus dem Krankenhaus entlassen werden $(6,0 \pm 1,2$ vs. 7,0 $\pm 1,5$ Tage, $p<0,01)$. Da während der Behandlung keine unerwünschten Nebenwirkungen auftraten, beurteilten die Autoren die Inhalationstherapie der Bronchiolitis mit vernebelter $3 \%$ iger Saline und Salbutamol als sicher und wirksam.

tr

Quelle: Luo et al. Pediatr Int 2010; 52(2):199-202

PNEUMONEWS

Herausgeber Klinik:

Prof. Dr. A. Gillissen, Kassel

Prof. Dr. M. J. Kohlhäufl, Stuttgart-

Gerlingen

\title{
Schriftleiter:
}

Priv.-Doz. Dr. S. Budweiser, Rosenheim (Beatmungsmedizin)

Priv.-Doz. Dr. G. Friedel, Stuttgart-Gerlingen (Thoraxchirurgie)

Prof. Dr. A. Ghofrani, Gießen, Dr. K. Gutjahr, Leipzig (Erkrankungen des Lungenkreislaufs)

Prof. Dr. A. Gillissen, Kassel (Asthma bronchiale)

Prof. Dr. M. Griese, München (Pädiatrische Pneumologie)

Prof. Dr. A. Günther, Prof. Dr. Ph. Markart, Gießen (Interstitielle Lungenerkrankungen)

Prof. Dr. M. J. Kohlhäufl, Stuttgart-Gerlingen (Tumoren)

Dr. S. R. Ott, Bern (Infektiologie)

Prof. Dr. K. Rasche, Wuppertal, Prof. Dr. K.-H. Rühle, Hagen, Prof. Dr. H. Schäfer, Völklingen (Schlafmedizin)

Prof. Dr. G. Rohde, Maastricht/Niederlande (COPD, Emphysem)

Dr. K. Schultz, Bad Reichenhall (Rehabilitation)

Prof. Dr. M. Schwab, Prof. Dr. K. Mörike, Tübingen (Pharmakologische Therapie)

\section{Redaktion:}

Dr. Beate Schumacher (Chefredaktion,

verantwortlich, Anschrift wie Verlag,

beate.schumacher@springer.com)

Ute Kempf (ute.kempf@springer.com),Dr. Judith

Neumaier (judith.neumaier@t-online.de), Dr.

Thomas Riedel, Angelika Holzgreve (Assistenz

Chefredaktion, angelika.holzgreve@springer. com)

Verlag:

Springer Medizin, (๑) Urban \& Vogel GmbH, Aschauer Str. 30, D-81549 München,

Tel.: o 89/20 30 43-13 00, Fax: -13 99,

http://www.urban-vogel.de.

Springer Medizin ist Teil der Fachverlagsgruppe Springer Science+Business Media.

Geschäftsführer:

Harm van Maanen, Dr. med. Dirk Einecke, Ulrich Huber, Stephan Kröck, , Dr. med. Esther Wieland Matthias Wissel.

Anzeigenverkauf:

Ines Spankau (Anzeigenleitung, -1339, verantwortlich, Anschrift wie Verlag), Anzeigenpreisliste Nr. 3 vom 1. Januar 2010

\section{Corporate Publishing:}

Dr. Ulrike Fortmüller (Leitung München, -1417)

Herstellung:

Matthias Wissel (Leitung); w\&Co MediaServices München $\mathrm{GmbH}$ \& Co KG (Layout); Dieter L.

Adam, München (Titelbild)

\section{Vertrieb/Marketing Services:}

Frank Niemann (Leitung, -14 11)

\section{Abonnement:}

Die Zeitschrift erscheint $6 \times$ jährlich. Neue Bezugspreise ab 2010: Einzelheft $12 €$ inkl. der deutschen MwSt. zuzügl. 2,50 € Versandkosten; Jahresabo $75 €$; Studenten/Ärzte in Aus- und Weiterbildung $60 €$, jeweils zuzügl. Versand (Inland 19,50 €, Ausland $35 €$ )

Bestellungen nimmt der Verlag und jede Buchhandlung entgegen. Das Abonnement verlängert sich jeweils um ein Jahr, wenn es nicht zwei Monate vor Ende des Bezugszeitraums abbestellt wurde.

\section{Abonnentenservice:}

Tel.: o 62 21/3 45-43 04; Fax: o 62 21/3 45-42 29

Gesamtherstellung: Stürtz $\mathrm{GmbH}$, AlfredNobel-Str. 33, D-97080 Würzburg

Originalien: Zur Veröffentlichung kommen nur Arbeiten, die an anderer Stelle weder angeboten noch erschienen sind. Die Autoren sind verpflichtet zu prüfen, ob Urheberrechte Dritter berührt werden. Alle CME-Manuskripte werden von unabhängigen Experten begutachtet. Eine redaktionelle Bearbeitung bleibt vorbehalten. Autoren, die mit vollem Namen genannt sind, veröffentlichen ihre Beiträge in alleiniger Verantwortung.

Copyright: Der Verlag behält sich das aus schließliche Recht der Verbreitung, Überset zung und jeglicher Wiedergabe auch von Teilen dieser Zeitschrift durch Nachdruck, Fotokopie, Mikrofilm, EDV-Verwertung, Funk- oder Fernsehaufzeichnung vor. Jede gewerblich hergestellte oder benutzte Fotokopie verpflichtet nach $\S 54$ (2) UrHG zur Gebührenzahlung an die VG Wort, Abt. Wissenschaft, Goethestr. 49, D-80336 München, von der die Modalitäten zu erfragen sind.

Rechtseinräumung der Autoren: Mit der Einsendung eines Manuskripts zur Veröffentlichung überträgt der Verfasser dem Verlag für den Fall der Annahme das Recht, das Manuskript geändert oder unverändert, ganz oder teilweise in Pneumo-News und anderen Publikationen der Fachverlagsgruppe, in den zugehörigen Onlinediensten, in Onlinedatenbanken Dritter und, soweit vereinbart, gegen Nachhonorar in Sonderdrucken für Industriekunden zu nutzen.

Hinweis: Die in dieser Zeitschrift angegebenen Dosierungen - vor allem von Neuzulassungen - sollten in jedem Fall mit dem Beipackzettel der verwendeten Medikamente verglichen werden.

ISSN 1865-5467

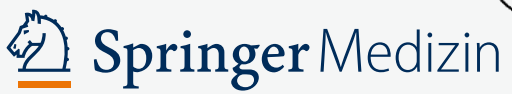

